\title{
A Family of Binary Approximating Subdivision Schemes based on Binomial Distribution
}

\author{
MUHAMMAD ASGHAR*, AND GHULAM MUSTAFA*
}

RECEIVED ON 22.01.2018 ACCEPTED ON 25.05.2018

\begin{abstract}
A simplest way is introduced to generate a generalized algorithm of univariate and bivariate subdivision schemes. This generalized algorithm is based on the symbol of uniform B-splines subdivision schemes and probability generating function of Binomial distribution. We present a family of binary approximating subdivision schemes which has maximum continuity and less support size. Our proposed family members $\mathbf{P}_{4}, \mathbf{P}_{5}, \mathbf{P}_{6}$, and $\mathbf{P}_{7}$, have $\mathbf{C}^{7}, \mathbf{C}^{9}, \mathbf{C}^{11}$ and $\mathrm{C}^{13}$ continuities respectively. In fact, we use Binomial probability distribution to increase the continuity of uniform B-splines subdivision schemes up to more than double. We present the complete analysis of one family member of proposed schemes and give a visual performance to check smoothness graphically. In our analysis, we present continuity, Holder regularity, degree of generation, degree of reproduction and limit stencils analysis of proposed family of subdivision schemes. We also present a survey of high continuity subdivision schemes. Comparison shows that our proposed family of subdivision schemes gives high continuity of subdivision schemes comparative to existing subdivision schemes. We have found that as complexity increases the continuity also increases. In the last, we give the general formula for tensor product surface subdivision schemes and also present the visual performance of proposed tensor product surface subdivision schemes.
\end{abstract}

Key Words: Binary,Approximating Subdivision Schemes,Uniform B-Splines,Binomial Distribution, Tensor Product.

\section{INTRODUCTION}

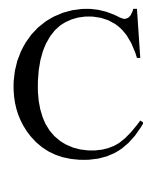

AGD (Computer Aided Geometric Design) is a field which is related to Applied Mathematics. In this arena, we follow procedures for the shapes that are usually used in the design, computer graphics for their mathematical study. The important tool of CAGD is subdivision schemes. Subdivision schemes are the iterative formulas which are used for generation of smooth curves and surfaces form initial polygon or initial mesh.
Developing new subdivision schemes for curve and surface design has its own importance. In the field of subdivision schemes de Rham [1] and Chaikin [2] are regarded as the pioneers. They developed the corner cutting schemes which generate $\mathrm{C}^{1}$ limit curve. Now a day wide variety of approximating and interpolating schemes have been proposed in the literature which possess shape parameters. Dyn et. al. [3] presented a

Authors E-Mail: (m.asgharmaths@gmail.com, ghulam.mustafa@iub.edu.pk)

* Department of Mathematics, The Islamia University of Bahawalpur, Bahawalpur, Pakistan.

This is an open access article published by Mehran University Research Journal of Engineering and Technology, Jamshoro under the CC by 4.0 International License. 
four-point subdivision scheme which has $\mathrm{C}^{2}$ continuity. Siddiqi and Ahmad [4-6] offered three, five and six points approximating subdivision schemes having $\mathrm{C}^{2}, \mathrm{C}^{4}$ and $\mathrm{C}^{6}$ limit curves respectively when the shape parameter $\mathrm{W}=1 / 4$.

Mustafa et. al. [7] presented 6-point approximating subdivision schemes which give $\mathrm{C}^{6}$ continuity when the shape parameter $w \in(-1.6,1.3)$. Mustafa et. al. [8] presented a generalized proof of the smoothness of 6-point interpolatory scheme. They proved that 6-point interpolatory scheme introduced by [8] have $\mathrm{C}^{3}$ continuity when the shape parameter $w \in[0.0139,0.0143]$. Ghaffar et. al. [9] presented 3-point approximating subdivision scheme which is $\mathrm{C}^{3}$ continuous at $\mu=1$. Ghaffar and Mustafa [10] presented a family of even point ternary approximating subdivision schemes. One family member of 6-point ternary approximating subdivision scheme gives $\mathrm{C}^{7}$ continuity at $w=1 / 12$. Tan et. al. [11] presented a new four-point shapepreserving subdivision scheme with two shape parameters which has $\mathrm{C}^{3}$ continuity at $\alpha=1, \beta=32$.

Hameed and Mustafa [12] presented a family of univariate and bivariate binary approximating subdivision schemes using Lane- Riesenfeld algorithm.

Zheng et. al. [13] presented designing multi-parameter curve subdivision schemes with high continuity. They offered a technique to increase the continuity of subdivision scheme. The way of increasing the continuity is multiplying the symbol with $(1+z / 2)^{k}$ factor, after multiplication they get $C^{n+k}$ continuous subdivision schemes. But the demerits of this technique are: when they multiply symbol with factor then complexity, support size and mathematical computation of subdivision schemes are also increased. After this literature, we offer a technique that gives high continuity subdivision schemes, less complexity and support size.
In this paper, we construct a parametric family of subdivision schemes which have high continuity, less complexity and support size. The construction of proposed family based on probability density function of binomial distribution and symbol of uniform B-splines subdivision schemes. We give the comparison of continuity analysis with uniform B-splines and other existing binary subdivision schemes which have high continuity. This paper is also a survey of high continuity subdivision schemes.

The paper is organized as follows. In Section 2, we present construction of a family of binary approximating subdivision schemes. Analysis of the proposed family is presented in Section 3. Section 4 is for general formulae of a family of tensor product surface subdivision schemes. Applications and conclusions are drawn in Sections 5 and 6 respectively.

\section{CONSTRUCTION OF A FAMILY OF SCHEMES}

In this section, we will present an algorithm for parametric families of subdivision schemes. There are two subsections, one is for a family of Binomial distribution based subdivision schemes and other is for a family of binary approximating subdivision schemes which is based on the symbol of uniform Bsplines and probability generating function of binomial distribution.

\subsection{Binomial Distribution Based Subdivision Schemes}

Here we will construct binary approximating subdivision schemes using the binomial distribution. The Binomial distribution is given by: 
$a_{i}=\left(\begin{array}{c}n \\ i\end{array}\right) p^{i}(1-p)^{n-i}, i=0,1, \ldots, n$

where the variable $i$ with $0 \leq i \leq n$ and the parameter $\mathrm{n}$ are any positive integers. The parameter $p$ is a probability of successive events; the range of $p$ is $0 \leq p \leq 1$. The distribution describes the probability of exactly $\bar{i}$ successes in $n$ (fixed number) trials if the probability of success in a single trial is $\mathrm{p}$ (we sometimes also use 1-p the probability for a failure). Bernoulli first presented in a work which was published in [14]. After substituting the different values of $n, n \geq 1$ in Equation (1), we get $(n+1)$ point binary approximating subdivision schemes. The general form of $(n+1)$-point binary approximating subdivision schemes is:

$$
\begin{aligned}
f_{2 i}^{k+1}= & a_{0} f_{i}^{k}+a_{1} f_{i+1}^{k}+\ldots++a_{n-1} f_{i+n-1}^{k}+a_{n} f_{i+n}^{k} \\
& f_{2 i+1}^{k+1}=a_{n} f_{i}^{k}+a_{n-1} f_{i+1}^{k}+\ldots++a_{1} f_{i+n-1}^{k}+a_{o} f_{i+n}^{k}
\end{aligned}
$$

For $n=1$ in Equation (1), we get $a_{0}=(1-p)$ and $a_{1}=p$ then Equation (2) takes the form:

$$
\begin{aligned}
& f_{2 i}^{k+1}=(1-p) f_{i}^{k}+p f_{i+1}^{k} \\
& f_{2 i+1}^{k+1}=p f_{i}^{k}+(1-p) f_{i+1}^{k}
\end{aligned}
$$

If we put $p=1 / 2$ in Equation (3), then we get a well-known Chaikin [2] corner cutting scheme. Similarly, for $n=2$, we get $a_{0}=(1-p)^{2}, a_{1}=2 p(1-p)$ and $a_{2}=p$ then Equation (2) becomes:

$$
\begin{aligned}
& f_{2 i}^{k+1}=(1-p)^{2} f_{i}^{k}+2 p(1-p) f_{i+1}^{k}+p^{2} f_{i+2}^{k} \\
& f_{2 i+1}^{k+1}=p^{2} f_{i}^{k}+2 p(1-p) f_{i+1}^{k}+(1-p)^{2} f_{i+2}^{k}
\end{aligned}
$$

We can generate a family of $(n+1)$-point binary approximating subdivision schemes by using the different values of $n$ and $p \in(0,1)$.

\subsection{Family of Binary Approximating Subdivision Schemes with High Continuity}

This subsection is for the general symbol of a family of binary approximating subdivision schemes with one shape parameter. Probability density function of binomial distribution and $(n+1)$ the degree polynomial uniform Bsplines for all $n \geq 1$ are used to construct the general symbol of the family of binary approximating subdivision schemes. The symbol of uniform B-splines [15] subdivision schemes are given as:

$A_{n}(z)=\frac{(1+z)^{n+1}}{2^{n}}$

The probability generating function of binomial distribution [16] is given as:

$$
B_{n}(z)=\sum_{i=0}^{n}\left(\begin{array}{c}
n \\
i
\end{array}\right) p^{i}(1-p)^{n-i} z^{i}
$$

The symbol defined in Equation (4) has $C^{n-1}$ continuous subdivision schemes. In order to increase the continuity of uniform B-splines and generate a new family of approximating subdivision schemes, we take a product of symbol of uniform B-splines schemes (4) and probability density function of the binomial distribution (5). The general symbol of a family of approximating subdivision schemes is defined as:

$P_{n}(z)=A_{n}(z) B_{n}(z)$

The simplest form of the symbol is:

$$
P_{n}(z)=\frac{(1-p)^{n-1}}{2^{n}} \sum_{i=0}^{n}\left(\begin{array}{c}
n \\
i
\end{array}\right) p^{i}(1-p)^{n-i} z^{i}
$$


This is the general formula for a family of binary approximating subdivision schemes with one parameter.

Our proposed family of binary approximating subdivision schemes with one parameter has high continuity, less support size and many existing subdivision schemes are particular cases of our proposed subdivision schemes. We get the family members of approximating subdivision schemes corresponding to each value of $n$ in Equation (6), and for different values of $p$ can generate the different subdivision schemes. Table 1 gives the complexity and mask of proposed family of approximating subdivision schemes.

\section{ANALYSIS OF THE SCHEMES}

This section aims to present the analysis of a proposed family of binary approximating subdivision schemes. Here we only present the analysis of one family member $\mathrm{P}_{2}$ of binary subdivision schemes, the analysis of other family members is similar.

\subsection{Convergence and Smoothness Analysis}

Continuity is an important property of subdivision schemes. Continuity of a subdivision scheme refers to the differentiability of the limit curve/surface produced by subdivision process. A high continuous subdivision scheme gives a more smooth limit curve. We use Laurent polynomial (symbol) method [17] to calculate integer class continuity of the $\mathrm{P}_{\mathrm{n}}$-schemes. In general $\mathrm{P}_{\mathrm{n}}(\mathrm{z})$ in Equation (6) is $\mathrm{C}$ continuous but when we substitute the value of $\mathrm{P}=1 / 2, \mathrm{P}_{\mathrm{n}}(\mathrm{z})$ have maximum continuity. Table 2 gives the comparison of continuity analysis of uniform B-splines schemes $A_{n}(z)$, proposed $P_{n}(z)$ schemes in general and proposed $\mathrm{P}_{n}(\mathrm{z})$ schemes at $\mathrm{p}=1 / 2$.

Theorem-1: The 3-point binary scheme corresponding to the symbol $\mathrm{P}_{2}$ is $\mathrm{C}^{2}$ continuous.

Proof: The Laurent polynomial of the 3-point binary scheme is obtained by substituting $n=2$ in Equation (6), which is:

$$
P_{2}(z)=\frac{1}{4}(1+z)^{3}\left((1-p)^{2}+2 p(1-p) z+p^{2} z^{2}\right)(7)
$$

TABLE 1. HERE WE PRESENT THE MASK OF FAMILY OF BINARY APPROXIMATING SUBDIVISION SCHEMES CORRESPONDING TO DIFFERENT VALUES OF $n$, HERE $m$ SHOWS COMPLEXITY OF THE SCHEMES (i.e. 2-3,....POINT SCHEMES)

\begin{tabular}{|c|c|c|}
\hline$n$ & $m$ & Mask \\
\hline 1 & 2 & $P_{1}=\frac{1}{2}[p, p+1,2-p, 1-p]$ \\
\hline 2 & 3 & $P_{2}=\frac{1}{4}\left[p^{2}, p^{2}+2 p, 1-2 p^{2}+4 p, 3-2 p^{2}, 3-4 p+p^{2}, 1-2 p+p^{2}\right]$ \\
\hline 3 & 4 & $\begin{array}{c}P_{3}=\frac{1}{8}\left[p^{3}, p^{3}+3 p^{2}+6 p^{2}+3 p-3 p^{3}, 1+9 p-3 p^{3}, 3 p^{3}+6 p+4-12 p^{2}\right. \\
\left.\quad 6-6 p+3 p^{3}-3 p^{2},-p^{3}+6 p^{2}-9 p+4,1-3 p-p^{3}+3 p^{2}\right]\end{array}$ \\
\hline 4 & 5 & $\begin{aligned} P_{4}= & \frac{1}{16}\left[p^{4}, p^{4}+4 p^{3},-4 p^{4}+8 p^{3}+6 p^{2}, 4 p-4 p^{4}-8 p^{3}+18 p^{2}, 16 p+6 p^{2}-24 p^{3}+6 p^{2}-24 p^{3}+6 p^{4}+1,6 p^{4}+5-30 p^{2}+20 p,\right. \\
& \left.10-30 p^{2}-4 p^{4}+24 p^{3}, 6 p^{2}-20 p+10-4 p^{3}, 18 p^{2}-8 p^{3}+5-16 p+p^{4}, 1-4 p-4 p^{3}+p^{4}+6 p^{2}\right]\end{aligned}$ \\
\hline
\end{tabular}


This implies

$$
P_{2}(z)=\left(\frac{1+z}{2}\right)^{2} b(z)
$$

where $b(z)=(1+z) c(z), c(z)=\left((1-p)^{2}+2 p(1-p) z+p^{2} z^{2}\right)$.

$$
\|c(z)\|_{\infty}=\max \left\{(1-p)^{2}|+| p^{2}|,| 2 p(1-p) \mid\right\}
$$

The condition for $\|c(z)\|_{\infty}<1$ is $0<\mathrm{p}<1$. Therefore by [13], if $\|c(z)\|_{\infty}<1$, then $\mathrm{c}(\mathrm{z})$ is contractive and $\mathrm{b}(\mathrm{z})$ is converges. If $b(z)$ is convergent then scheme corresponding to $\mathrm{P}_{2}(\mathrm{z})$ is $\mathrm{C}^{2}$ continuous.
HolderRegularityAnalysis: Holder regularity is an extension of convergence and continuity which gives more information about any scheme. Lower and upper bounds on Holder continuity are calculated by using Rioul's method [18]. Moreover, exact upper bounds on Holder continuity can also be derived by using Floater and Muntingh algorithm [19]. In Table 3, we summarized the results of lower and upper bounds of Holder continuity which depends on the value of $p$. Moreover, we obtain exact Holder regularity when $\mathrm{p}=1 / 2$.

Theorem-2: Holder regularity of 3-point binary scheme corresponding to the symbol $\mathrm{P}_{2}(\mathrm{z})$ is 3 at $\mathrm{p}=1 / 2$.

TABLE 2. HERE WE SHOW THE COMPARISON OF CONTINUITY ANALYSIS OF UNIFORM B-SPLINES SCHEMES CORRESPONDING TO $\mathrm{A}_{\mathrm{n}}(\mathrm{z})$, PROPOSED SCHEMES CORRESPONDING TO $\mathrm{P}_{\mathrm{n}}(\mathrm{z})$ IN GENERAL AND PROPOSED SCHEMES CORRESPONDING TO $P_{n}(z)$ at $p=1 / 2$ FOR DIFFERENT $n$

\begin{tabular}{|c|c|c|c|}
\hline $\mathrm{n}$ & $\mathrm{A}_{\mathrm{n}}(\mathrm{z}$ & $\mathrm{P}_{\mathrm{n}}(\mathrm{z})$ & $\mathrm{P}_{\mathrm{n}}(\mathrm{z})$ at $\mathrm{W}=1 / 2$ \\
\hline 1 & $\mathrm{C}_{0}$ & $\mathrm{C}_{1}$ & $\mathrm{C}_{2}$ \\
\hline 2 & $\mathrm{C}_{1}$ & $\mathrm{C}_{2}$ & $\mathrm{C}_{3}$ \\
\hline 3 & $\mathrm{C}_{2}$ & $\mathrm{C}_{3}$ & $\mathrm{C}_{5}$ \\
\hline 4 & $\mathrm{C}_{3}$ & $\mathrm{C}_{4}$ & $\mathrm{C}_{7}$ \\
\hline 5 & $\mathrm{C}_{4}$ & $\mathrm{C}_{5}$ & $\mathrm{C}_{9}$ \\
\hline 6 & $\mathrm{C}_{5}$ & $\mathrm{C}_{6}$ & $\mathrm{C}_{11}$ \\
\hline 7 & $\mathrm{C}_{6}$ & $\mathrm{C}_{7}$ & $\mathrm{C}_{13}$ \\
\hline
\end{tabular}

TABLE 3. HERE WE PRESENT THE HOLDER REGULARITY ANALYSIS OF SOME MEMBERS OF THE FAMILY OF BINARY $P_{n}$ SCHEMES FOR $n=1,2$, AND 3. HERE $n, k, L_{h}, U_{h}$ AND $E_{h}$ ARE ANY POSITIVE INTEGER, COMMON FACTORS, LOWER BOUND OF HOLDER REGULARITY, THE UPPER BOUND OF HOLDER REGULARITY AND EXACT HOLDER REGULARITY AT

\begin{tabular}{|c|c|c|c|c|}
\hline$n$ & $k$ & $\mathrm{~L}_{\mathrm{h}}$ & $\mathrm{U}_{\mathrm{h}}$ & $\mathrm{E}_{\mathrm{h}}$ \\
\hline 1 & 2 & $\left\{\begin{array}{ccc}2(1-p)^{2} & \text { if } & 0<p<0.5 \\
2 p & \text { if } & 0.5<p<1\end{array}\right\}$ & $\left\{\begin{array}{cll}2(1-p) & \text { if } & 0<p<0.5 \\
2 p & \text { if } & 0.5<p<1\end{array}\right\}$ & 2 \\
\hline 2 & 3 & $\left\{\begin{array}{ccc}2(1-p)^{2} & \text { if } & 0 \leq p \leq 0.3 \\
2 p(1-p) & \text { if } & 0.3 \leq p \leq 0.6 \\
2 p^{2} & \text { if } & 0.6 \leq p \leq 0.9\end{array}\right\}$ & $\left\{2 p^{2}+2(p-1)^{2}\right\}$ & 3 \\
\hline 3 & 4 & $\left\{\begin{array}{ccc}\beta_{1} & \text { if } & 0<p<0.3 \\
\beta_{2} & \text { if } & 0.3<p \leq 0.7 \\
\beta_{3} & \text { if } & 0.7<p<1\end{array}\right\}$ & $\left\{\begin{array}{lll}\beta_{4} & \text { if } & 0<p \leq 0.5 \\
\beta_{5} & \text { if } & 0.5<p<1\end{array}\right\}$ & 6 \\
\hline
\end{tabular}
$\mathrm{p}=1 / 2$

Mehran University Research Journal of Engineering \& Technology, Volume 38, No. 4, October, 2019 [p-ISSN: 0254-7821, e-ISSN: 2413-7219] 
Thus the limit stencil of scheme corresponding to $\mathrm{P}_{2}(\mathrm{z})$ is given by $P_{2}=\left\{l, l_{1}, l_{2}, l_{3}\right\}$. Which complete the proof.

\subsection{Survey and Comparison with Existing Schemes}

Here we will present a survey of high continuity parametric subdivision schemes. We also show that our proposed family of binary approximating subdivision schemes gives the highest continuity as compare to existing parametric subdivision schemes. Table 5 present a survey and comparison with proposed family of approximating subdivision schemes.

\section{TENSOR PRODUCT FAMILY OF APPROXIMATING SUBDIVISION SCHEMES}

In this section, we will present the generalized formulae for a family of approximating tensor product surface subdivision schemes. Uniform B-splines subdivision schemes for tensor product surface is defined as:

$$
A_{n}\left(z_{1}, z_{2}\right)=\frac{\left(1+z_{1}\right)^{n+1}\left(1+z_{2}\right)^{n+1}}{2^{2 n}}
$$

Probability density function of Binomial distribution for tensor product surfaces subdivision schemes is defined as:

$$
B_{n}\left(z_{1}, z_{2}\right)=\sum_{i=0}^{n}\left(\begin{array}{l}
n \\
i
\end{array}\right) p^{i}(1-p)^{n-i} z_{1}^{i} \sum_{i=0}^{n}\left(\begin{array}{l}
n \\
i
\end{array}\right) p^{i}(1-p)^{n-i} z_{2}^{i}
$$

The general symbol of a family of approximating subdivision schemes is defined as:

$\mathrm{P}_{\mathrm{n}}\left(\mathrm{z}_{1}, \mathrm{z}_{2}\right)=\mathrm{A}_{\mathrm{n}}\left(\mathrm{z}_{1}, \mathrm{z}_{2}\right) \mathrm{B}_{\mathrm{n}}\left(\mathrm{z}_{1}, \mathrm{z}_{2}\right)$

which is the general formula for tensor product of a proposed family of approximating subdivision schemes, where $A_{n}\left(z_{1}, z_{2}\right)$ is defined in Equation (11) and $B_{n}\left(z_{1}, z_{2}\right)$ is defined in Equation (12) with $p \in(0,1)$. By substituting the different values of $n$ in Equation (13), we can obtain the family members of tensor product surface subdivision schemes.

TABLE 5. SHOWS THE COMPARISON OF CONTINUITY ANALYSIS OF EXISTING PARAMETRIC SUBDIVISION SCHEMES AND PROPOSED FAMILY OF SCHEMES E,C AND $P_{n}$ DENOTE THE EXISTING SCHEMES, CONTINUITY OF EXISTING SCHEMES AND CONTINUITY OF PROPOSED SCHEMES At $p=1 / 2$ RESPECTIVELY

\begin{tabular}{|c|c|c|}
\hline $\mathrm{E}$ & $\mathrm{C}$ & $\mathrm{P}^{\mathrm{n}}$ \\
\hline 3-Point [4] & $\mathrm{C}^{2}$ at $\mathrm{w}=1$ & $\mathrm{C}^{3}$ \\
\hline 3-Point [9] & $\mathrm{C}^{3}$ at $\mu=1$ & $\mathrm{C}^{3}$ \\
\hline 4-Point [3] & $\mathrm{C}^{2}$ & $\mathrm{C}^{5}$ \\
\hline 4-Point [21] & $\mathrm{C}^{4}$ & $\mathrm{C}^{5}$ \\
\hline 4-Point [22] & $\mathrm{C}^{5}$ at $\mathrm{w}=1 / 4$ & $\mathrm{C}^{7}$ \\
\hline 5-Point [5] & $\mathrm{C}^{4}$ at $\mathrm{w}=1 / 4$ & $\mathrm{C}^{7}$ \\
\hline 5-Point [23] & $\mathrm{C}^{5}$ at $\mathrm{w}=1 / 128$ & $\mathrm{C}^{7}$ \\
\hline 5-Point [11] & $\mathrm{C}^{3}$ at $\alpha=1, \beta=32$ & $\mathrm{C}^{9}$ \\
\hline 6-Point [4] & $\mathrm{C}^{4}$ at $\mathrm{w}=1 / 4$ & $\mathrm{C}^{9}$ \\
\hline 6-Point [6] & $\mathrm{C}^{9}$ with 6 paprameter & $\mathrm{C}^{9}$ \\
\hline 6-Point [24] & $\mathrm{C}^{7}$ at $w \in=1 / 12$ & $\mathrm{C}^{9}$ \\
\hline 6-Point [10] & $\mathrm{C}_{8}$ if $w \in(-1.6,1.3)$ & $\mathrm{C}^{9}$ \\
\hline 6-Point [7] & $\mathrm{C}^{3}$ if $\left.w 0.0139,0.0143\right]$ & $\mathrm{C}^{9}$ \\
\hline 6-Point [8] & $\mathrm{C}^{2}$ & $\mathrm{C}^{9}$ \\
\hline 6-Point [25] & $\mathrm{C}^{2}$ & $\mathrm{C}^{11}$ \\
\hline 6-Point [26] & $\mathrm{C}^{9}$ & \\
\hline
\end{tabular}

Mehran University Research Journal of Engineering \& Technology, Volume 38, No. 4, October, 2019 [p-ISSN: 0254-7821, e-ISSN: 2413-7219] 
Proof: From Equation (7) we have $b_{0}=(1-p)^{2}, b_{1}=4 p(1-p)$, $\mathrm{b}_{0}=2 \mathrm{p}^{2}, \mathrm{k}=3, \mathrm{~m}=2$ and $\mathrm{b}=2$ thus $\mathrm{q}=0,1,2, \mathrm{~B}_{\mathrm{o}}, \mathrm{B}_{1}$ and $\mathrm{B}_{2}$ are the matrices with elements

$\left(B_{0}\right)_{i, j}=b_{2+i-2 j},\left(B_{1}\right)_{i, j}=b_{3+i-2 j}$, and $\left(B_{2}\right)_{i, j}=b_{4+i-2 j}$

Where $\mathrm{i}, \mathrm{j}=1$ and 2 . Thus $\mathrm{B}_{0}, \mathrm{~B}_{1}$ and $\mathrm{B}_{2}$ are given by:

$B_{0}\left(\begin{array}{cc}b_{1} & 0 \\ b_{2} & b_{0}\end{array}\right), B_{1}\left(\begin{array}{cc}b_{2} & b_{0} \\ 0 & b_{1}\end{array}\right)$, and $B_{2}\left(\begin{array}{cc}0 & b_{1} \\ 0 & b_{2}\end{array}\right)$

By [14], the Holder regularity is given by $r=k-\log _{b}(\mu)$, where $\mu$ is the joint spectral radius of the matrices $B_{0}, B_{1}$ and $B_{2}$ that is $\mu=\rho\left(B_{0}, B_{1}, B_{2}\right)$. For bounds on Holder regularity, we calculate

$\max \left\{\rho\left(B_{0}\right), \rho\left(B_{1}\right), \rho\left(B_{2}\right)\right\} \leq \mu \leq \max \left\{\left\|B_{0}\right\|_{\infty},\left\|B_{1}\right\|_{\infty},\left\|B_{2}\right\|_{\infty}\right\}$

Since $\mu$ is bounded from below by the spectral radii and from above by the norm of the metrics $\mathrm{B}_{0}, \mathrm{~B}_{1}$, and $\mathrm{B}_{2}$. We have

$\max \left\{2(1-p)^{2}, 4 p(1-p), 2 p^{2}\right\} \leq \mu \leq \max \left\{4 p(1-p), 2 p^{2}+2(p-1)^{2}, 2 p^{2}\right\}$

The value of $\mu$ depends on the range of $w$.

$$
\left\{\begin{array}{ccc}
2(1-p)^{2} & \text { if } & 0 \leq p \leq 0.3 \\
4 p(1-p) & \text { if } & 0.3 \leq p \leq 0.6 \\
2 p^{2} & \text { if } & 0.6 \leq p \leq 0.9
\end{array}\right\} \leq \mu \leq\left\{2 p^{2}+2(p-1)^{2}\right\}
$$

By using Equation (9), we can calculate the lower and upper bounds of Holder regularity for different values of p. The exact Holder regularity of 3-point binary scheme corresponding to the symbol $\mathrm{P}_{2}(\mathrm{z})$ is $\mathrm{r}=3-\log _{2}(1)=3$ at $\mathrm{p}=1 / 2$, which completes the proof.

where

$$
\begin{aligned}
& \beta_{1}=2-6 p+6 p^{2}-2 p^{3}, \beta_{2}=2 p(1-p)\left(-\frac{3}{2}+\frac{1}{2} \sqrt{9+32 p^{2}-32 p}\right) \\
& \beta_{3}=2 p(1-p)\left(-\frac{3}{2}+\frac{1}{2} \sqrt{9+32 p^{2}-32 p}\right), \beta_{4}=6\left|p^{2}(1-p)\right|+2\left|(p-1)^{3}\right|
\end{aligned}
$$

and

$$
\beta_{5}=6\left|p(1-p)^{2}\right|+2\left|p^{3}\right|
$$

\subsection{Generation and Reproduction Analysis}

The subdivision scheme with symbol $\mathrm{P}_{\mathrm{n}}(\mathrm{z})$ reproduces polynomials of degree $d$ with respect to the parameterizations with $\tau=p_{n}^{\prime}(1) / 2$ if and only if

$$
p_{n}^{k}(-1)=0 \text { and } p_{n}^{k}(1)=2 \prod_{j=0}^{k-1}(\tau-j), \mathrm{k}=0,1,2, \ldots, \mathrm{d}(10)
$$

Polynomial reproduction of degree $d$ requires the polynomial generation of degree $d$ [13]. Support of the schemes is calculated by [20]. Table 4 summarizes the results of support, generation degree, reproduction degree, shift parameter and parametrization of proposed schemes corresponding to $\mathrm{P}_{\mathrm{n}}(\mathrm{z})$ and generation degree at $\mathrm{p}=1 / 2$ of proposed schemes corresponding to $\mathrm{P}_{\mathrm{n}}(\mathrm{z})$.

Theorem-3: Generation degree of 3-point binary scheme

\begin{tabular}{|c|c|c|c|c|c|c|}
\hline $\mathrm{n}$ & $\mathrm{S}$ & $\mathrm{G}_{\mathrm{d}}$ & $\mathrm{R}_{\mathrm{d}}$ & $\tau$ & $\mathrm{P}$ & $\mathrm{G}_{\mathrm{d}}$ at $\mathrm{p}=1 / 2$ \\
\hline 1 & 3 & 1 & 1 & $\tau=1$ & \multirow{4}{*}{ Prima/Dual } & 2 \\
\hline 2 & 5 & 2 & 1 & $\tau=(4 p+3) / 2$ & & 4 \\
\hline 3 & 7 & 3 & 1 & $\tau=2+3 p$ & & 6 \\
\hline 4 & 9 & 4 & 1 & $\tau=(8 p+5) / 2$ & & 9 \\
\hline
\end{tabular}
corresponding to the symbol $\mathrm{P}_{2}(\mathrm{z})$ is 2 .

TABLE 4. HERE WE PRESENT AN ANALYSIS OF SOME MEMBERS OF THE FAMILY OF BINARY $P_{n}$ SCHEMES FOR $n=1,2,3$ AND $4_{m}$ HERE $n, S, G_{d}, R_{d}, \tau$ AND PARE ANY POSITIVE INTEGER, SUPPORT, GENERATION DEGREE, REPRODUCTION DEGREE, SHIFT PARAMETER, AND PARAMETRIZATION 
Proof: The Laurent polynomial of 3-point binary scheme defined in Equation (7) can be written as:

$$
P_{2}(z)=\left(\frac{1+z}{2}\right)^{2+1} b(z)
$$

where $b(z)=2\left((1-p)^{2}+2 p(1-p) z+p^{2} z^{2}\right)$.

Hence generation degree is 2 .

Theorem-4: The 3-point binary scheme corresponding to the symbol $\mathrm{P}_{2}(\mathrm{z})$ reproduces polynomial of degree 1 concerning the dual parameterizations and also gives the primal parametrization at $p=1 / 4$

Proof: By taking the first derivative of Equation (7) and put $\mathrm{z}=1$, we get $\left(\mathrm{P}_{2}\right)^{\prime}(1)=4 \mathrm{p}+3$ This implies that $\tau=4 \mathrm{p}+3$ / 2 , so the scheme corresponding to the symbol $\mathrm{P}_{2}(\mathrm{z})$ has dual parametrization. This scheme also gives the primal parametrization at $p=1 / 4$. We can easily verify that the first and second derivatives of $\mathrm{P}_{2}(\mathrm{z})$ if $\mathrm{z}=-1$ are equal to 0 . Further, we can also verify (10) for $\mathrm{k}=0$ and 1 . This completes the proof.

\subsection{Limit Stencil Analysis}

The limit stencil is a way to obtain a point on the limit curve in the form of the original control points.

The procedure for calculating the limit stencils is presented in [17]. Here we present the analysis of limit stencils of one family member.

Theorem-4: Limit stencil of 3-point binary scheme corresponding to the symbol $\mathrm{P}_{2}(\mathrm{z})$ is $\mathrm{P}_{2}=\left\{l, l_{1}, l_{2}, l_{3}\right\}$, where

$l=\frac{(5 p+1) p^{3}}{6\left(p^{2}-p+2\right)}$ $l_{1}=\frac{\left(5 p^{3}+11 p^{2}-18 p-4\right) p}{6\left(p^{2}-p+2\right)}$

$l_{2}=\frac{4 p^{4}-31 p^{3}+45 p^{2}-13 p-6}{6\left(p^{2}-p+2\right)}$

and

$l_{3}=\frac{\left(4 p^{2}-11 p+6\right)\left(p^{2}-2 p+1\right)}{6\left(p^{2}-p+2\right)}$

Proof: The subdivision matrix of the scheme corresponding to the symbol $\mathrm{P}_{2}(\mathrm{z})$ defined by Equation (7) is:

$$
A=\left(\begin{array}{cccc}
\frac{p^{2}}{4}+\frac{p}{2} & -\frac{p^{2}}{2}+\frac{3}{4} & \frac{p^{2}}{4}-\frac{p}{2}+\frac{1}{4} & 0 \\
\frac{p^{2}}{4} & -\frac{p^{2}}{2}+p+\frac{1}{4} & -\frac{p^{2}}{4}-p+\frac{3}{4} & 0 \\
0 & \frac{p^{2}}{4}+\frac{p}{2} & -\frac{p^{2}}{2}+\frac{3}{4} & \frac{p^{2}}{4}-\frac{p}{2}+\frac{1}{4} \\
0 & \frac{p^{2}}{4} & -\frac{p^{2}}{2}+p+\frac{1}{4} & \frac{p^{2}}{4}-p+\frac{3}{4}
\end{array}\right)
$$

Eigenvalues of A are $\lambda_{0}=1, \lambda_{1}=1 / 2, \lambda_{2}=1 / 4$ and $\lambda_{3}=-\mathrm{p}^{2} / 2$ $+p / 2$. The matrix of eigenvectors corresponding to eigenvalues is:

$$
B=\left(\begin{array}{cccc}
1 & \frac{4 p-5}{1+4 p} & \frac{7 p^{2}-16 p+9}{7(2+7 p)} & \frac{\left(p^{2}-2 p+1\right)\left(p^{4}-5 p^{3}+6 p-4\right)}{p^{3}\left(-3 p-p^{2}-1+p^{3}\right)} \\
1 & -\frac{4 p-3}{1+4 p} & \frac{7 p^{2}-10 p+3}{p(2+7 p)} & -\frac{\left(p^{2}-2 p+1\right)\left(p^{2}-5 p+4\right)}{p\left(-3 p-p^{2}-1+p^{3}\right)} \\
1 & \frac{4 p-1}{1+4 p} & \frac{7 p-4}{p(2+7 p)} & -\frac{\left(p^{2}-2 p+1\right)(3+p)}{-3 p-p^{2}-1+p^{3}} \\
1 & 1 & 1 & 1
\end{array}\right)
$$

We can define the diagonal matrix $\mathrm{D}$ as:

$$
D=\left(\begin{array}{cccc}
1 & 0 & 0 & 0 \\
0 & \frac{1}{2} & 0 & 0 \\
0 & 0 & \frac{1}{4} & 0 \\
0 & 0 & 0 & -\frac{p^{2}}{2}+\frac{p}{2}
\end{array}\right)
$$


By diagonalization of the matrix $\mathrm{A}$, we get $\mathrm{A}+\mathrm{BDB}^{-1}$, where

$$
B=\left(\begin{array}{cccc}
\frac{p^{3}(5 p+1)}{6\left(p^{2}-p+2\right)} & -\frac{p\left(5 p^{3}+11 p^{2}-18 p-4\right)}{6\left(p^{2}-p+2\right)} & c & g \\
\frac{p^{3}(4 p+1)}{2\left(p^{2}-p+2\right)} & -\frac{p\left(p^{2}+2 p-2\right)(1+4 p)}{2\left(p^{2}-p+2\right)} & d & h \\
\frac{p^{3}(7 p+2)}{3\left(2 p^{2}-2 p+1\right)} & -\frac{p\left(p^{2}+2 p-1\right)(2+7 p)}{3\left(2 p^{2}-2 p+1\right)} & e & i \\
a & b & f & j
\end{array}\right)
$$

Where

$$
\begin{aligned}
& a=\frac{p^{3}\left(-3 p-p^{2}-1+p^{3}\right)}{2\left(2 p^{6}-6 p^{5}-16 p^{3}+14 p^{2}+13 p^{4}-7 p+2\right)} \\
& c=-\frac{5 p^{4}-31 p^{3}+45 p^{2}-13 p-6}{2\left(p^{2}-p+2\right)} \\
& b=-\frac{3 p^{3}\left(-3 p-p^{2}-1+p^{3}\right)}{2\left(2 p^{6}-6 p^{5}-16 p^{3}+14 p^{2}+13 p^{4}-7 p+2\right)} \\
& g=-\frac{\left(5 p^{2}-11 p+6\right)\left(p^{2}-2 p+1\right)}{6\left(p^{2}-p+2\right)} \\
& d=-\frac{\left(p^{2}-2 p+1\right)(p-1)(1+4 p)}{2\left(p^{2}-p+2\right)} \\
& h=-\frac{\left(p^{2}-2 p+1\right)(p-1)(1+4 p)}{2\left(p^{2}-p+2\right)} \\
& f=-\frac{3 p^{3}\left(-3 p-p^{2}+1+p^{3}\right)}{2\left(2 p^{6}-6 p^{5}-16 p^{3}=14 p^{2}-7 p+2\right)} \\
& e=-\frac{p(2+7 p)\left(p^{2}-4 p+2\right)}{3\left(2 p^{2}-2 p+1\right)} \\
& j=-\frac{p^{3}\left(-3 p-p^{2}-1+p^{3}\right)}{2\left(2 p^{6}-6 p^{5}-16 p^{3}+14 p^{2}+13 p^{4}-7 p+2\right)}
\end{aligned}
$$

$i=-\frac{p(2+7 p)\left(p^{2}-2 p+1\right)}{3\left(2 p^{2}-2 p+1\right)}$

Since $\mathrm{A}=\mathrm{BDB}^{-1}$ this implies $\mathrm{A}^{\mathrm{j}}=\mathrm{BD}^{\mathrm{j}} \mathrm{B}^{-1}$,

Where

$$
D^{j}=\left(\begin{array}{cccc}
1^{j} & 0 & 0 & 0 \\
0 & 0.5^{j} & 0 & 0 \\
0 & 0 & 0.25^{j} & 0 \\
0 & 0 & 0 & \left(-\frac{p^{2}}{2}+\frac{p}{2}\right)^{j}
\end{array}\right)
$$

Therefore

$$
D^{\infty}=\lim _{j \rightarrow \infty} D^{j}=\left(\begin{array}{cccc}
0 & 0 & 0 & 0 \\
0 & 0 & 0 & 0 \\
0 & 0 & 0 & 0 \\
0 & 0 & 0 & 0
\end{array}\right)
$$

By [17], we have

$$
\left(\begin{array}{l}
f_{-2}^{\infty} \\
f_{-1}^{\infty} \\
f_{0}^{\infty} \\
f_{1}^{\infty}
\end{array}\right)=\left(\begin{array}{llll}
l & l_{1} & l_{2} & l_{3} \\
l & l_{1} & l_{2} & l_{3} \\
l & l_{1} & l_{2} & l_{3} \\
l & l_{1} & l_{2} & l_{3}
\end{array}\right)\left(\begin{array}{l}
f_{-2}^{\infty} \\
f_{-1}^{0} \\
f_{0}^{0} \\
f_{1}^{0}
\end{array}\right)
$$

Where

$$
\begin{aligned}
& l=\frac{(5 p+1) p^{3}}{6\left(p^{2}-p+2\right)} \\
& l_{1}=-\frac{\left(5 p^{3}+11 p^{2}-18 p-4\right) p}{6\left(p^{2}-p+2\right)} \\
& l_{2}=-\frac{5 p^{4}-31 p^{3}+45 p^{2}-13 p-6}{6\left(p^{2}-p+2\right)}
\end{aligned}
$$

and

$$
l_{3}=-\frac{\left(5 p^{2}-11 p+6\right)\left(p^{2}-2 p+1\right)}{6\left(p^{2}-p+2\right)}
$$




\section{APPLICATIONS}

In this section, we will show the visual performance of proposed schemes. These examples show smooth curves which pass through a set of given points. The control polygons are drawn by a dashed line and the smooth curves obtained by our proposed schemes by a full line.
Fig. 1(a-c) is an example of limit curves for open and closed polygons, Fig. 2(a-f) present limit curves for close polygons and Fig. 3(a-c) give the comparison of limit curves generated by the schemes corresponding to $\mathrm{P}_{2}, \mathrm{P}_{3}$ and $\mathrm{P}_{4}$. After comparison, we see that $\mathrm{P}_{4}$ gives more smooth curves as compare to $\mathrm{P}_{2}+$ and $\mathrm{P}_{3}$ at $\mathrm{p}=1 / 2$ Figs. 4-5(a-e) are the visual performances of surface subdivision schemes $\mathrm{P}_{3}$.

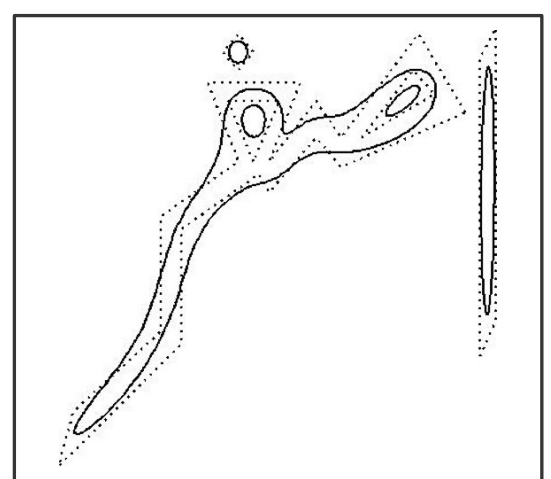

(a)

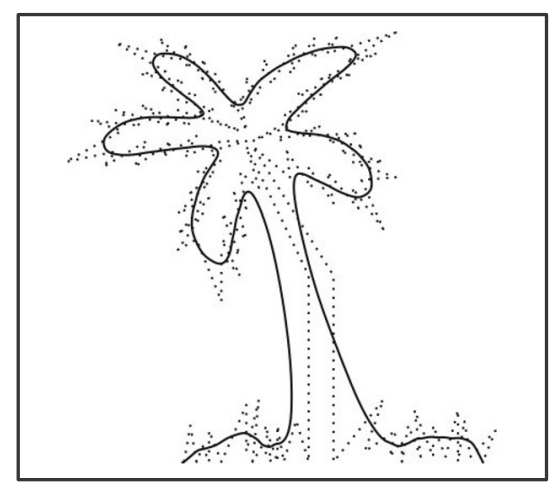

(b)

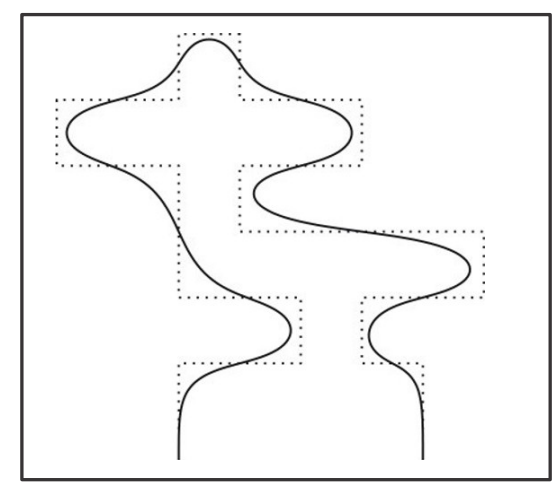

(c)

FIG. 1. PRESENT LIMIT CURVES FOR OPEN AND CLOSED POLYGONS PRODUCED BY A SCHEME CORRESPONDING TO P 2

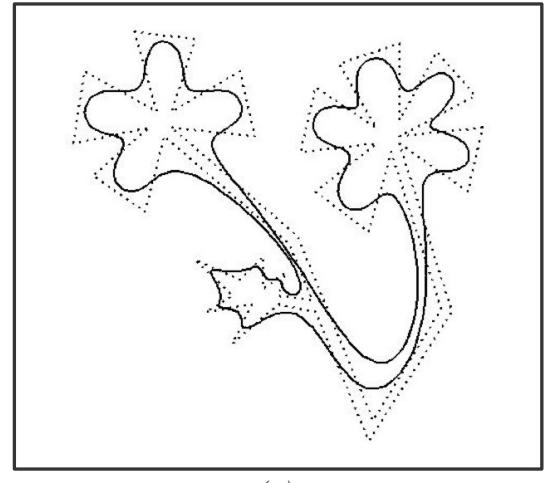

(a)

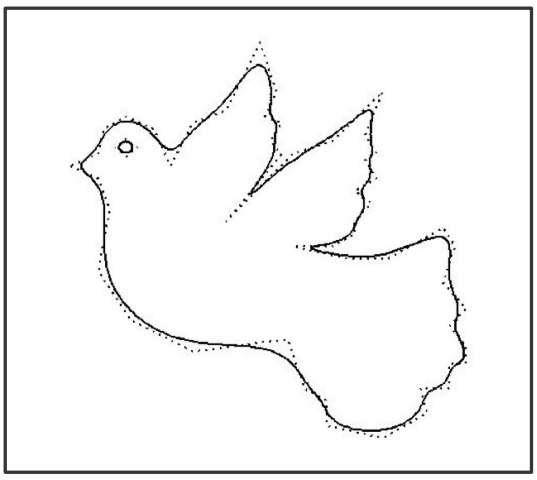

(d)

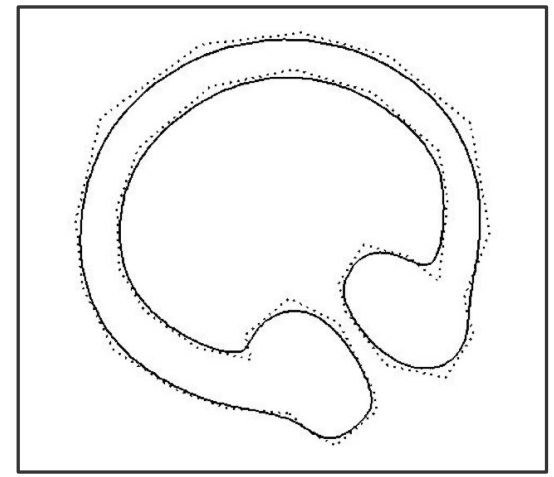

(b)

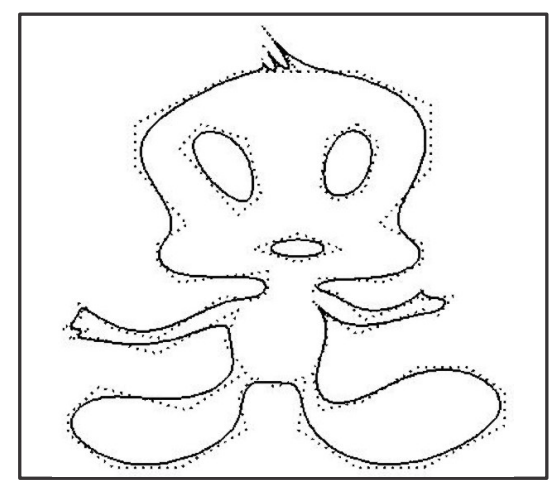

(e)

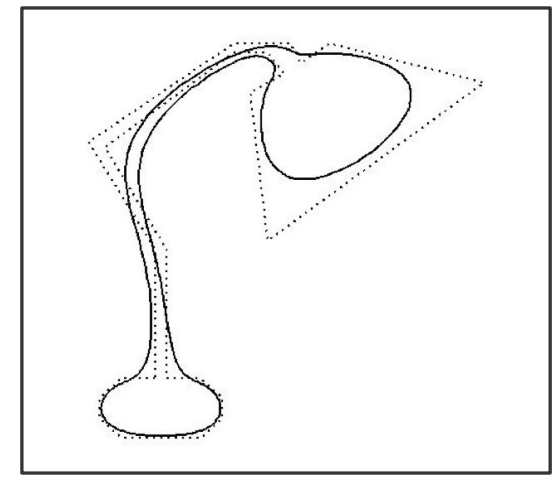

(c)

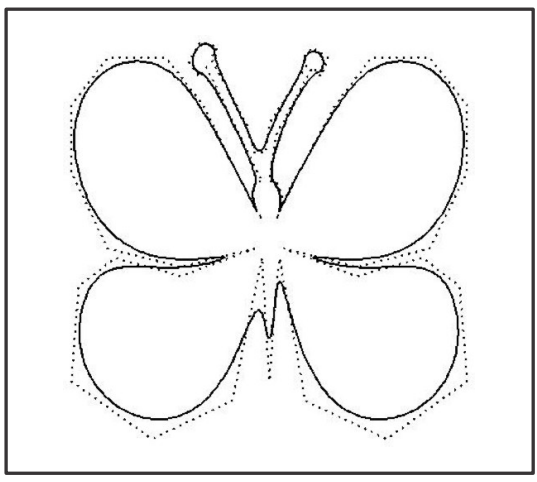

(f)

FIG. 2. PRESENT LIMIT CURVES FOR CLOSE POLYGONS PRODUCED BY A SCHEME CORRESPONDING TO P, 


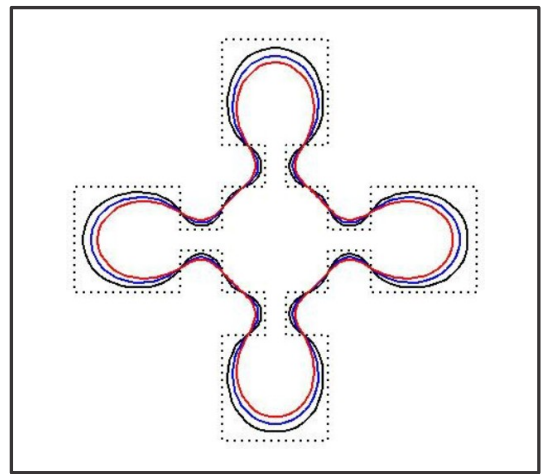

(a)

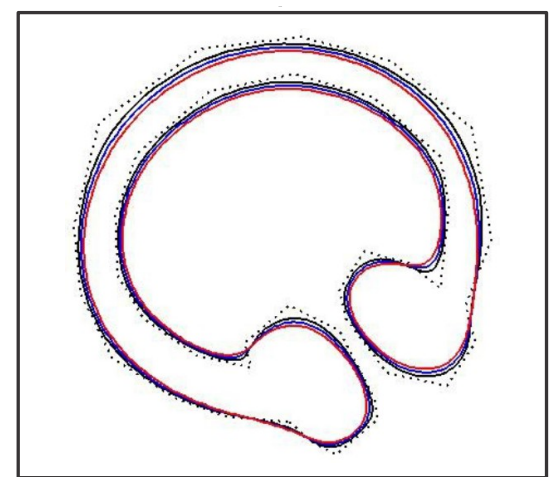

(b)

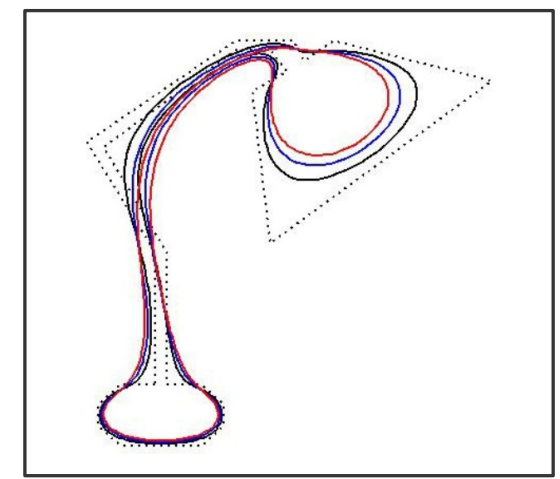

(c)

FIG. 3. PRESENT COMPARISON OF LIMIT CURVES FOR CLOSE POLYGONS PRODUCED BY A SCHEME CORRESPONDING TO P, $P$, AND $P_{4}$. HERE BLACK DOTTED LINES SHOWS THE INITIAL POLYGON, BLACK SOLID LINE IS THE LIMIT CURVE GENERATED BY A SCHEME CORRESPONDING TO P, THE BLUE SOLID LINE IS THE LIMIT CURVE GENERATED BY A SCHEME CORRESPONDING TO $P_{3}$ AND RED SOLID LINE IS THE LIMIT CURVE GENERATED BY A SCHEME CORRESPONDING TO $P_{4}$

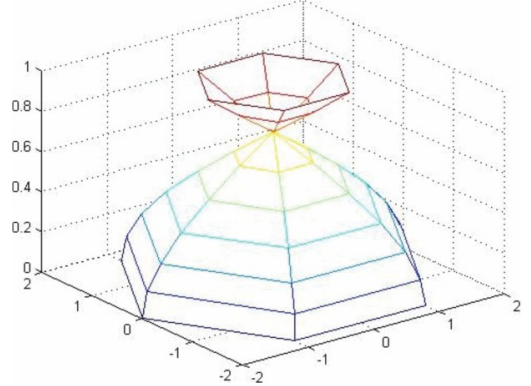

(a) INITIAL SURFACE

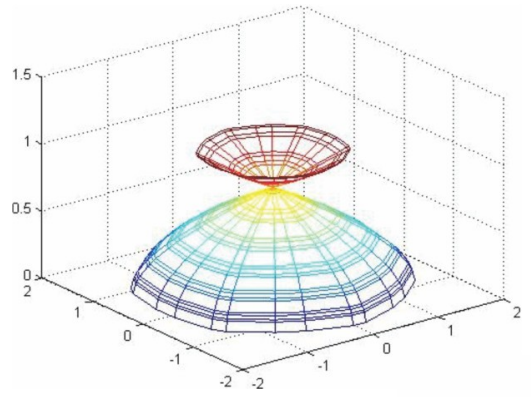

(c) LEVEL 2

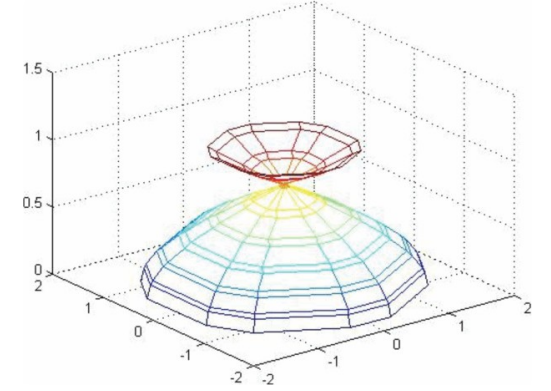

(b) LEVEL 1

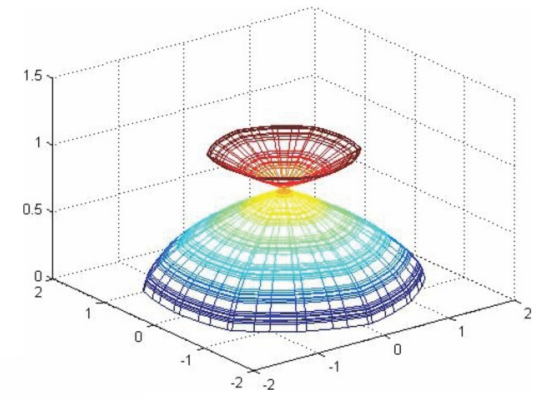

(d) LEVEL 3

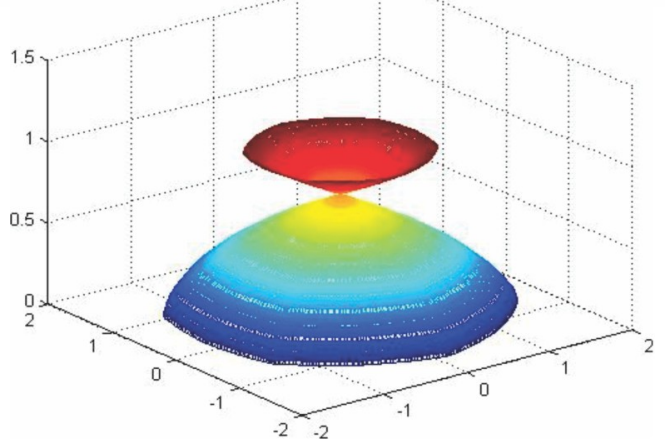

(e) LIMIT SURFACE

FIG. 4. PRESENT LIMIT CURVES FOR CLOSE POLYGONS PRODUCED BY A SCHEME CORRESPONDING TO P ${ }_{3}$ SURFACES SUBDIVISION SCHEME

Mehran University Research Journal of Engineering \& Technology, Volume 38, No. 4, October, 2019 [p-ISSN: 0254-7821, e-ISSN: 2413-7219] 


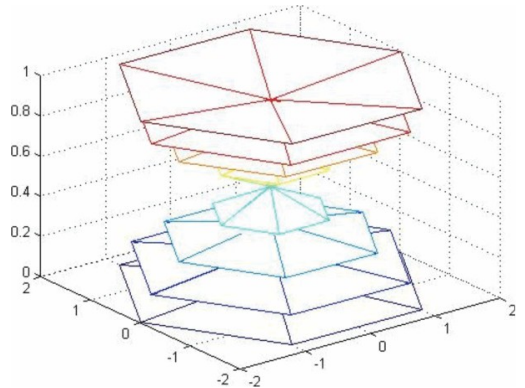

(a) INITIAL SURFACE

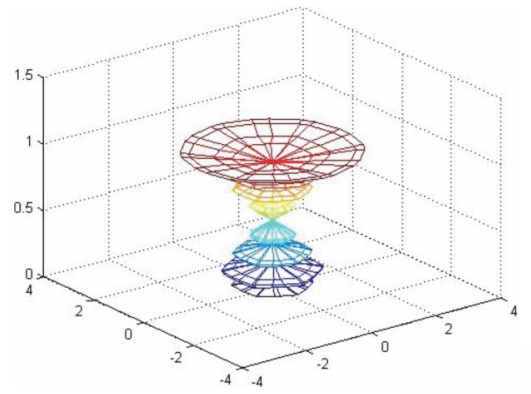

(c) LEVEL 2

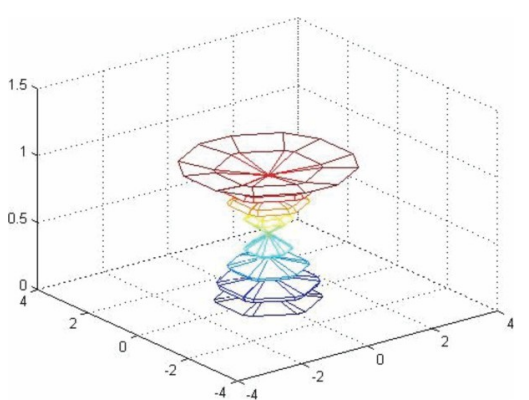

(b) LEVEL 1

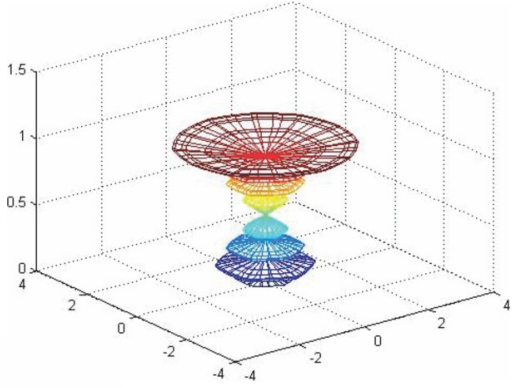

(d) LEVEL 3

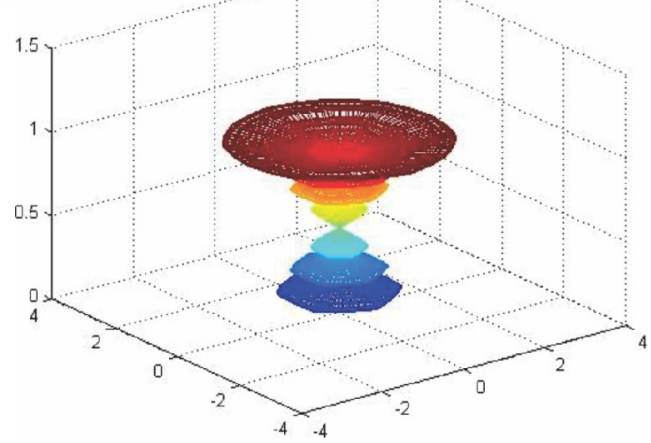

(e) LIMIT SURFACE

FIG. 5. PRESENT LIMIT CURVES FOR CLOSE POLYGONS PRODUCED BY A SCHEME CORRESPONDING TO P ${ }_{3}$ SURFACES SUBDIVISION SCHEME

\section{CONCLUSION}

In this paper, we have presented a generalized algorithm to introduce a parametric family of binary approximating subdivision schemes with high continuity. In our proposed algorithm, the continuity of uniform B-splines subdivision schemes increased up to more than double. We also give the complete analysis of one family member of proposed schemes. Visual performance shows the smoothness of a family of schemes graphically. Tensor product surface formula of proposed family of schemes is also presented. This paper also contains a survey of high continuity parametric subdivision schemes.

\section{ACKNOWLEDGEMENT}

This work is supported by NRPU (National Research Program for Universities), No. 3183, Pakistan.

\section{REFERENCES}

[1] Rham, de. G., "Un Peu De Mathématiques à Propos D'uneCourbe Plane", Elemente Der Mathematik, Volume 2, pp. 73-76, 1947.

[2] Chaikin, G.M., “An Algorithm for High-Speed Curve Generation", Computer Graphics and Image Processing, Volume 3, No.4, pp. 346-349, 1974. 
[3] Dyn, N., Floater, M.S., and Hormann, K., "A C² 4-Point Subdivision Scheme with Fourth Order Accuracy and Its Extensions", Mathematical Methods for Curves and Surfaces: Troms2004, Nashboro Press, Brentwood, Volume 128, No. 1, pp. 145-156, 2005.

[4] Siddiqi, S.S., and Ahmad, N., "A New 3-Point Approximating $\mathrm{C}^{2}$ Subdivision Scheme", Applied Mathematics Letters, Volume 20, No. 6, pp. 707-711, 2007.

Siddiqi, S.S., and Ahmad, N., "A New 5-Point Approximating Subdivision Scheme", International Journal of Computer Mathematics, Volume 85, No. 1, pp. $65-72,2008$.

[6] Siddiqi, S.S., and Ahmad, N., "A C ${ }^{6}$ Approximating Subdivision Scheme", Applied Mathematics Letters, Volume 21, No. 7, pp. 722-728, 2008.

Mustafa, G., Khan, F., and Ghaffar, A., "The $m$-Point Approximating Subdivision Scheme", Lobachevskii Journal of Mathematics, Volume 30, No. 2, pp. 138-145, 2009

[8] Mustafa, G., Khan, F., and Hashmi, M.S., “A Generalized Proof of the Smoothness of 6-Point Interpolatory Scheme", Journal of Information and Computing Science, Volume 5, No. 4, pp. 299-304, 2010.

[9] Ghaffar, A., Mustafa, G. and Qin, K., "Unification and Application of 3-Point Approximating Subdivision Schemes of Varying Arity", Open Journal of Applied Sciences, Volume 2, No. 4, pp. 48-52, 2013.

[10] Ghaffar, A., and Mustafa, G., "A Family of Even-Point Ternary Approximating Schemes", ISRN Applied Mathematics, Article ID 197383, pp. 14, 2012.

[11] Tan, J., Zhuang, X., and Zhang, L., "A New 4-Point Shape-Preserving $\mathrm{C}^{3}$ Subdivision Scheme", Computer Aided Geometric Design, Volume 31, No. 1, pp. 57-62, 2014.

[12] Hameed, R., and Mustafa, G., "Construction and Analysis of Binary Subdivision Schemes for Curves and Surfaces Originated from Chaikin Points", International Journal of Analysis, Article ID 1092476, pp. 15, 2016.
[13] Zheng, H.C., Huang, S.P., Guo, F., and Peng, G.H., "Designing Multi-Parameter Curve Subdivision Schemes with High Continuity", Applied Mathematics and Computation, Volume 243, pp. 197-208, 2014.

[14] Bernoulli, J., "ArsConjectandi", Opus Posthumum. Accedit Tractatus de Seriebusinfinitis, et Epistola Gallicescripta de ludo Pilaerecticularis. Impensis Thurnisiorum, Fratrum, Basel, 2013.

[15] Hormann, K., and Sabin, M.A., "A Family of Subdivision Schemes with Cubic Precision", Computer Aided Geometric Design, Volume 25, No. 1, pp. 41-52, 2008.

[16] Walck, C., "Handbook on Statistical Distributions for Experimentalists", University of Stockholm, 2007.

[17] Hormann, K., "Subdivision Schemes for Geometric Modeling a Hands-on Tutorial", Dolomites Research Notes on Approximation, [ISSN 2035-6803], University of Verona, 2012.

[18] Rioul, O., "Simple Regularity Criteria for Subdivision Schemes", SIAM Journal on Mathematical Analysis, Volume 23, No. 6, pp. 1544-1576, 1992.

[19] Floater, M.S., and Muntingh, G., "Exact Regularity of Symmetric Univariate Subdivision Schemes, Geometry Seminar", Centre of Mathematics for Applications, University of Oslow, Norway, 2012.

[20] Ivrissimtzis, I.P., Sabin, M.A., and Dodgson, N.A., “On the Support of Recursive Subdivision", ACM Transactions on Graphics, Volume 23, No. 4, pp. 1043-1060, 2004.

[21] Hongxing, Z., "A Class of 4-Point Subdivision Schemes with Two Parameters and its Properties", Journal of Computer Aided Design and Computer Graphics, Volume 16, No. 8, pp. 1140-1145, 2004.

[22] Ghaffar, A., Mustafa, G., and Qin, K., "The 4-Point aAry Approximating Subdivision Scheme", Open Journal of Applied Sciences, Volume 3, No. 1, pp. 106-111, 2013. 
[23] Cao, H., and Tan, J., “A Binary 5-Point Relaxation Subdivision Scheme”, Journal of Information and Computational Science, Volume 10, No. 18 , pp. 5903-5910, 2013.

[24] Hao, Y.X., Wang, R.H. and Li, C.J., "Analysis of A 6Point Binary Subdivision Scheme", Applied Mathematics and Computation, Volume 218, No. 7, pp. 3209-3216, 2011 .
[25] Romani, L., "From Approximating Subdivision Schemes for Exponential Splines to High-Performance Interpolating Algorithms", Journal of Computational and Applied Mathematics, Volume 224, No. 1, pp. 383-396, 2009.

[26] Ko, K.P., Lee, B.G. and Yoon, G.J., "A Study on The Mask of Interpolatory Symmetric Subdivision Schemes", Applied Mathematics and Computation, Volume 187, No. 2, pp. 609-621, 2007. 